\title{
SEGUNDA OPINIÃO ESPECIALIZADA EDUCACIONAL NO TELESSAÚDE BRASIL: UMA REVISÃO
}

\section{SPECIALIZED FORMATIVE SECOND OPINION IN BRAZIL TELEHEALTH: A REVIEW}

\author{
Edna Zakrzevski Padilha, Fabrício Rutz da Silva, Felipe Simão Pontes \\ Universidade Estadual de Campinas - Faculdade de Odontologia de Piracicaba (FOP-UNI- \\ CAMP). E-mail: ednapadilha@hotmail.com \\ Data de recebimento: 03/04/2013 \\ Data da aprovação: 18/05/2013
}

\section{RESUMO}

Este artigo explora a utilização da Telessaúde em relação às ações de segunda opinião especializada educacional no Brasil. As Tecnologias da Informação e Comunicação (TICs) estão sendo aproveitadas por toda a sociedade, e há um interesse crescente nas formas como elas podem ser usadas para atender e apoiar a saúde dos pacientes e as necessidades das comunidades. O objetivo da revisão da literatura foi de buscar as informações disponíveis a partir de pesquisas publicadas nas principais bases de dados eletrônicas (Lilacs, PubMed/Medline, Scielo) com especial referência para as ações de segunda opinião especializada educacional. A evidência sugere que as ações contribuíram principalmente para a descentralização do atendimento, garantindo maior abrangência e acesso à saúde em lugares mais distantes.

Palavras-chave: Telessaúde. Segunda opinião especializada educacional. Brasil

\begin{abstract}
This paper explores the use of telehealth in relation to the actions of the specialized formative second opinion in Brazil. Information and Communication Technologies (ICT) are being harnessed throughout society, and there is a growing interest in the ways in which they can be used to meet and support patients' health needs in the community. The aim of the literature review was to gather the information available from published research in the main electronic databases (Lilacs, PubMed/Medline, Scielo) with special reference to the actions of the specialized formative second opinion. The evidence suggests that actions contributed mainly to the decentralization of services ensuring greater access to health coverage in distant places.
\end{abstract}

Keywords: Telehealth. Specialized formative second opinion. Brazil

\section{Introdução}

Através de uma observação diagnóstica da realidade, percebe-se que o Sistema Único de Saúde - SUS não consegue uma resolubilidade para todos os problemas de saúde dos pacientes que necessitam de atendimento na rede de atenção básica. Portanto, o SUS está dividido hierarquicamente para prestar atendimentos em níveis primários, secundários e terciários, sendo que muitos pacientes são encaminhados para centros de referência. A demanda por atendimentos mais complexos tem feito com que haja demora no agendamento dos procedimentos necessários e consequente demora no tratamento de saúde.

O país possui um território grande com uma população relativamente dispersa, em regiões 
do interior ou isolada, e há uma discrepância na distribuição de profissionais e serviços especializados. Levanta-se um questionamento de como se poderia realizar a prestação de serviços especializados em tempo hábil para as populações que estão longe dos centros de referência sem que os pacientes necessitassem se deslocar de um local para outro. Outro item é o de como facilitar o acesso à educação permanente para os clínicos, levando-se em consideração as necessidades regionais.

As Tecnologias da Informação e Comunicação (TICs) estão sendo aproveitadas por toda a sociedade, e há um interesse crescente nas formas como elas podem ser usadas para atender e apoiar a saúde dos pacientes e as necessidades das comunidades. Mas, embora sejam utilizadas em larga escala no Brasil, existe pouca informação a respeito de sua aplicação na Telessaúde. O uso desta tecnologia em saúde tem o potencial de proporcionar benefícios tanto para o paciente e para o profissional, como para o país (SOIREFMANN et al., 2008).

Assim, o objetivo deste estudo é conhecer as aplicações e possíveis contribuições do Programa Telessaúde, através das ações de segunda opinião especializada educacional, já realizadas no Brasil.

\section{Materiais E Métodos}

Primeiro, foi realizada revisão da literatura com foco na descrição de Telessaúde e de segunda opinião especializada educacional. Após, foi realizada revisão da literatura dos últimos cinco anos nas principais bases de dados eletrônicas (Lilacs, PubMed/Medline, Scielo) com objetivo exploratório buscando levantar estudos sobre ações de segunda opinião especializada educacional relacionadas à aplicação do Programa Telessaúde Brasil. A busca nas bases de dados foi realizada entre abril e setembro de 2011. Nessas bases, utilizou-se para a busca a combinação de termos: telessaúde/telehealth, telemedicina/telemedicine, segunda opinião/second opinion e brasil/brazil.

$\mathrm{Na}$ busca foram encontrados 47 artigos. Os fatores para inclusão do texto no estudo foram: apresentar coleta de dados nacionais; ter sido publicado nos últimos cinco anos; apresentar conteúdo referente à segunda opinião formativa ou segunda opinião especializada educacional ou telediagnóstico ou teleconsulta com fins de segunda opinião.
Dos artigos, foram verificados que apenas 11 obedeciam aos critérios de inclusão; desses, 7 estavam publicados em periódicos nacionais. Os resultados foram analisados considerando as características dos estudos, e então descritos.

\section{Telessaúde}

Ambos os termos telessaúde e telemedicina referem-se ao uso das tecnologias de informação e da comunicação para fornecer serviços de saúde a distância (WEN, 2008). De acordo com a Organização Mundial de Saúde (2011), telemedicina é a oferta dos serviços de saúde por profissionais utilizando tecnologias de informação e comunicação onde a distância é um fator crítico. Através das tecnologias, é realizado o intercâmbio de informações válidas para diagnósticos, tratamentos e prevenção de doenças e lesões, pesquisa e avaliação, assim como para a educação continuada dos prestadores de cuidados, todos, no interesse da promoção da saúde dos indivíduos e suas comunidades.

De acordo com Wen (2008), as atividades da telemedicina podem ser divididas em três grandes conjuntos:

1. Teleducação Interativa e Rede de Aprendizagem Colaborativa: terminologia que designa o uso de tecnologias interativas para ampliar as possibilidades de construção de conhecimentos, de maneira que se aumentem as facilidades de acesso a materiais educacionais de qualidade e permita-se acesso a centros de referência ou à estruturação de novas sistemáticas educacionais (através de educação a distância ou por meio de tecnologias de apoio à educação presencial).

2. Teleassistência/Regulação e Vigilância Epidemiológica: são atividades desenvolvidas com fins assistenciais a distância, tais como a segunda opinião especializada. Sistemas podem ser desenvolvidos para permitir a integração de atividades assistenciais com educação, vigilância epidemiológica e gestão de processos em saúde.

3. Pesquisa Multicêntrica/Colaboração de Centros de Excelência e da Rede de "Teleciência": é a integração de diversos centros de pesquisa, permitindo a otimização de tempo e de custos, por meio do compartilhamento de dados, da capacitação e da padronização de métodos.

No âmbito do Ministério da Saúde do Brasil, através da Portaria ${ }^{\circ}$ 561, de 16 de março de 2006, é instituída a Comissão Permanente de Telessaúde. 
Mais tarde, considerando a necessidade de promover a integração entre as instituições capazes de desenvolver ações em saúde através de telemedicina e telessaúde, a Portaria $n^{0} 35$, de 04 de janeiro de 2007, instituiu o Programa Nacional de Telessaúde, definindo critérios para instalação de 900 pontos referentes ao Projeto Piloto Nacional de Telessaúde aplicada à atenção básica. Os resultados desse projeto auxiliaram para que fosse implantado o Programa Telessaúde Brasil pela Portaria $\mathrm{n}^{\mathrm{0}} 402$, de 24 de janeiro de 2010.

É colocado como propósito do programa Telessaúde Brasil a estruturação de uma rede de instituições parceiras, denominada Rede Telessaúde Brasil, com a implantação dos Núcleos Universitários de Telessaúde, dos Pontos de Telessaúde e dos Pontos Avançados de Telessaúde para apoio à Estratégia de Saúde da Família no Sistema Único de Saúde. O Núcleo Universitário de Telessaúde deve ser preferencialmente constituído em universidade pública e vinculado aos cursos de graduação da área da saúde; o Ponto de Telessaúde é indicado para ser implementado em Unidade de Saúde da Família; e o Ponto Avançado de Telessaúde, implementado em Escola Técnica do Sistema Único de Saúde (SUS) ou em serviço de saúde onde se realizem atividades de formação e educação permanente em saúde. No web site http:// www.telessaudebrasil.org.br podem ser encontradas informações mais detalhadas.

A telessaúde é considerada uma grande inovação tecnológica no nível social e cultural e que tem o potencial de aumentar o acesso e a qualidade dos serviços de saúde e diminuir os gastos do Sistema Único de Saúde (BRASIL, 2011).

No Brasil, devido ao extenso território e à má distribuição de recursos, as vantagens da telemedicina podem ser muito grandes. Entre elas, pode-se citar a facilitação do acesso a protocolos sistematizados, à educação a distância, à pesquisa colaborativa entre centros de ensino, sessões de segunda opinião, além da melhor assistência à população, principalmente em regiões remotas ou deficientes, onde o acesso aos serviços médicos é precário. A distância entre o tempo de diagnóstico e o tratamento diminui, o que aumenta a eficiência dos serviços médicos, justificando o investimento em tecnologia (LIMA et al., 2007).

Assim, a introdução da Telessaúde como uma ferramenta para apoiar a prestação dos serviços de saúde implica inúmeras mudanças para prestadores de serviços, organizações e o sistema de saúde como um todo, aspectos esses que devem ser considerados durante o processo de execução (BARROS, CARDOSO, 2008).

Para Wen e Haddad (2011), ao se falar da telemedicina e telessaúde como estratégia para melhorar a eficiência de um sistema de saúde e de todos os benefícios que elas podem gerar, deve-se também lembrar que as novas tecnologias por si só não são resolutivas. Ou seja, elas atingem o máximo de seu potencial quando os recursos humanos estão comprometidos no seu uso, havendo uma integração efetiva entre as instituições colaboradoras, no sentido de se conseguir o máximo de resultados.

\section{Segunda Opinião Especializada Educacional}

Para Wen $(2008,2011)$, a segunda opinião especializada educacional ou também chamada de segunda opinião especializada formativa são ações envolvendo um profissional a distância utilizando recursos de informática ou telecomunicação para transformar sua experiência em conhecimento para outro profissional. Essa é uma estratégia que permite o desenvolvimento de um enfoque educacional baseado na problemática real, de maneira a atender as necessidades específicas dos profissionais de cada região. Assim há uma orientação contextualizada para a tomada de decisão, e o profissional especializado transmite conhecimentos fornecendo subsídios para a formação de raciocínio do profissional solicitante.

De acordo com o artigo $1^{\circ}$, parágrafo único da Portaria $\mathrm{n}^{\circ} 402$, de 24 de fevereiro de 2010:

[...] considera-se "Segunda Opinião Formativa" a resposta estruturada e sistematizada às perguntas formuladas pelas Equipes de Saúde da Família, a partir dos Pontos de Telessaúde e dos Pontos Avançados do Telessaúde, sobre temas relacionados ao diagnóstico, planejamento e execução de ações, individuais e coletivas, sobre processo de trabalho ou vinculados a casos clínicos atendidos nas Unidades de Saúde da Família. (BRASIL, 2010).

Barbosa et al. (2010) fazem uma diferenciação entre o telediagnóstico e a segunda opinião. Para eles, o sistema de telediagnóstico permite que os médicos em locais distantes e de poucos recursos possam enviar informações de seu paciente solicitando para médicos especialistas o diagnóstico. Por sua vez, o médico especialista acessa a base de dados, avalia os dados do paciente e emite o seu parecer. E então, o 
médico que solicitou o pedido pode acessar o diagnóstico. A diferença colocada é que no serviço de segunda opinião os médicos especialistas discutirão a respeito de casos de pacientes, sendo esses advindos do serviço de telediagnóstico ou criando seus próprios casos. Geralmente são colocados em discussão casos de difícil diagnóstico ou que englobem mais de uma especialidade médica. Um médico especialista, ao requisitar os serviços de segunda opinião, poderá escolher entre os médicos participantes da rede de cooperação.

Em particular, a teleconsulta é um processo assistencial, no qual o paciente é atendido por um ou mais médicos a distância. Neste serviço, o paciente tem acesso a profissionais de saúde de outras localidades ou especialidades através de uma sessão de conferência bidirecional multimídia (troca de áudio, vídeo e dados). A consulta ou ambulatório ocorre entre dois sites remotos visando à assistência ao paciente. Em um site, encontra-se o paciente acompanhado por seu médico assistente ou enfermeira e, em outro site, o(s) médico(s) consultor(es). Neste processo, é possível transmitir, a qualquer distância, além do áudio e vídeo dos participantes da sessão, dados demográficos ou clínicos do paciente (histórico clínico, exames de imagens, etc.). (TIS, 2011).

$\mathrm{Na}$ análise da literatura e com base no que foi exposto até aqui, ficou evidente que não há padronização dos termos, e os conceitos diferem para cada autor. Para fins metodológicos, neste estudo será considerada "segunda opinião especializada educacional" os termos: segunda opinião formativa, segunda opinião especializada educacional, telediagnóstico e teleconsulta desde que sejam com fins de solicitação de opinião de outro profissional.
Ferramentas tecnológicas têm sido desenvolvidas especialmente para que os integrantes das equipes de Saúde da Família encaminhem aos especialistas as dúvidas que surgirem durante a prática profissional. Para permitir a troca de informações entre os pontos remotos, são citadas duas maneiras distintas de sincronia, ou seja, da forma pela qual a transmissão dos dados e a recepção são feitas. Uma maneira diz respeito ao formato assíncrono, podendo ser também mencionada como store-and-forward (armazenamento e envio), em off-line e transmissão remota, em que os usuários não necessitam estar conectados ao mesmo tempo. A outra maneira é em formato síncrono, em real-time (tempo real) ou online, na qual é preciso que os usuários estejam conectados ao mesmo momento. A utilização de um ou outro sistema dependerá da análise adequada da situação e qual o problema a ser enfrentado (MIOT, PAIXÃO, WEN; 2005).

\section{Resultados}

A busca demonstrou uma incipiente, porém crescente literatura a respeito do assunto, visto que se trata de um emprego relativamente recente da tecnologia, embora se mostre um terreno fértil e propício à expansão e, portanto, ao lançamento de artigos e estudos científicos.

A tabela 1 resume os 11 artigos que obedeciam aos critérios de inclusão e, portanto, foram selecionados para compor este estudo. Observou-se que, desses, 6 apresentaram características com fins de repassar dados referentes à implantação do sistema/ serviço, e os outros 5 estudos apresentaram preocupação com relação à qualidade da decisão diagnóstica em segunda opinião.

Tabela 1 - Resumo dos estudos incluídos na revisão

\begin{tabular}{|c|c|c|}
\hline Artigo & Especialidade Clínica & Forma de Utilização da Tecnologia \\
\hline Barbosa et al. 2010 & Multiespecialidades & Síncrona/Assíncrona \\
\hline Monteiro et al.. 2010 & Cardiologia & Assíncrona \\
\hline Ribeiro et al. 2010 & Cardiologia & Síncrona/Assíncrona \\
\hline Cardoso et al. 2010 & Cardiologia & Assíncrona \\
\hline Machado et al. 2010 & Multiespecialidades & Síncrona \\
\hline Hadad et al. 2010 & Multiespecialidades & Síncrona/Assíncrona \\
\hline Neira et al. 2010 & Radiologia & Assíncrona \\
\hline Silva et al. 2009 & Dermatologia & Assíncrona \\
\hline Trindade et al. 2008 & Dermatologia & Assíncrona \\
\hline Ribas et al. 2010 & Dermatologia & Assíncrona \\
\hline Torres-Pereira et al. 2008 & Odontologia & Assíncrona \\
\hline
\end{tabular}

Fonte: Elaborada pelos autores 


\section{Implantação De Sistemas De Segunda Opinião}

Barbosa et al. (2010) apresentaram o aplicativo HealthNet, o qual foi desenvolvido como suporte ao telediagnóstico e à segunda opinião médica. $\mathrm{O}$ HealthNet foi indicado com o propósito de melhorar a prestação de serviços de saúde em áreas distantes e carentes. Além disso, poderia permitir implantar um processo de cooperação médica entre grandes centros especialistas, como compartilhamento entre instituições participantes de informações clínicas do paciente, permitindo acesso rápido e completo aos dados e ao histórico da saúde do paciente, possibilitando uma intervenção mais segura e adequada para cada indivíduo.

Por sua vez, Monteiro et al. (2010) apresentaram o desenvolvimento de um protótipo de um aplicativo para sistematização de um modelo de segunda opinião médica para diagnóstico via eletrocardiograma. Através do software de segunda opinião médica, o médico responsável poderia gravar, enviar o traçado de eletrocardiograma junto às observações clínicas no aplicativo ao médico solicitado, que poderia registrar a segunda opinião e então devolver ao médico responsável. $\mathrm{O}$ aplicativo foi desenvolvido visando a sua utilização em ambulatórios médicos de universidades, mas também podendo ter outras aplicações, como em hospitais, postos de saúde, clínicas particulares e em universidades para educação continuada, além de monitoramento em domicílio. Os autores referem que o aplicativo foi criado visando a segurança e o sigilo das informações assim como a possibilidade de redução de custos, pois apresenta abrangência de atendimento às camadas sociais desfavorecidas, menor deslocamento de especialistas para regiões remotas e com isso a diminuição do ônus à saúde pública, além de inclusão social.

Em outra pesquisa, Ribeiro et al. (2010) analisaram a implantação de um programa de telecardiologia remoto no estado de Minas Gerais - Projeto Minas Telecardio, no qual as avaliações de Eletrocardiografia digital- ECG foram realizadas nas cidades e emitidas através da internet para a análise de uma equipe médica. Participaram do projeto 42.664 pacientes, com um total de 62.865 avaliações de ECG executadas. Foram realizadas 720 teleconsultas com 420 casos clínicos discutidos durante as atividades de telecardiologia e 300 teleconsultas em off-line. Os profissionais de saúde adaptaram-se rapidamente à ECG digital e às ferramentas do sistema e indicaram a oportunidade de alcançar um diagnóstico precoce, a discussão de casos clínicos e a educação continuada como as principais vantagens do projeto. De acordo com a percepção deles, o projeto resultou em uma redução de $70 \%$ no encaminhamento de pacientes para avaliação em centros de referência.

Para investigar os fatores associados com a implementação deste projeto, Cardoso et al. (2010) analisaram os 393 municípios do estado de Minas Gerais que eram candidatos a tal proposta. Os municípios foram divididos em dois grupos, o de intervenção $(n=82)$ - locais onde foram implementados os programas de telecardiologia - e o grupo comparativo $(\mathrm{n}=311)$ - todos os demais municípios que poderiam ser elegíveis. Foram comparados entre os dois grupos de municípios os índices demográficos, sociais e de desenvolvimento, de estrutura de cuidados em saúde e de mortalidade. Foi demonstrado que o sistema de cardiologia remoto foi implementado em municípios com menos necessidade de saúde e com os melhores índices de responsabilidade social. $\mathrm{O}$ índice de responsabilidade social reflete o sucesso escolar, cuidados de saúde, a segurança pública, emprego e renda; administração política; habitação; meio ambiente; cultura, lazer e esporte. Este melhor índice indica maior engajamento político e social municipal sendo, portanto, a governabilidade o fator que pode ter melhor influenciado para a implementação.

Machado et al. (2010) relataram a experiência de trabalho interdisciplinar com o sistema de telemedicina que permitiu a troca simultânea de vídeo e áudio em tempo real. O estudo mostra que, em uma unidade básica de saúde, de uma comunidade ribeirinha da Amazônia, das 80 consultas realizadas, 34 foram teleconsultas e/ou segunda opinião médica. A telemedicina possibilitou o contato direto e simultâneo com centros avançados em saúde, revertendo em melhoria da orientação de suporte à vida frente a situações de risco, melhoria da resolubilidade do atendimento primário e secundário e o acompanhamento por especialista, uma vez que o atendimento secundário era realizado apenas na zona urbana.

Fechando a análise quanto à implantação dos sistemas, Hadad et al. (2010) estudaram o envolvimento dos médicos quanto ao uso de teleconsulta 
online ou off-line em três dimensões: organização, individual e tecnologia. A análise apontou que a teleconsulta não tinha sido ainda totalmente compreendida pelos profissionais, sendo que a teleconsulta off-line foi indicada como uma solução para sanar dúvidas, sem a exposição do profissional. A falta de espaço físico e a falta de prática com informática apareciam como fatores negativos. Apesar das barreiras culturais, organizacionais e tecnológicas, a teleconsulta foi reconhecida por sua capacidade de produzir benefícios para o praticante, o paciente e para melhorar a qualidade dos serviços de cuidados primários em geral.

\section{Qualidade Da Decisão Diagnóstica Em Segunda Opinião}

Para a revisão de estudos qualitativos de diagnóstico, foram selecionados cinco trabalhos. No primeiro deles, Neira et al. (2010) avaliaram o processo de segunda opinião médica remota na especialidade de radiologia, através de 1.704 respostas de 34 médicos do Departamento de Diagnóstico por Imagem da Universidade Federal de São Paulo. Os dados indicaram que em $733(43,0 \%)$ das respostas o médico consultor teve "Confiança acima de 75\%" no seu diagnóstico, em 864 (50,7\%) o médico apresentou "Confiança abaixo de 75\%" no seu diagnóstico e 107 $(6,3 \%)$ não responderam. Conseguiram verificar também que $65 \%$ das respostas dos questionários indicaram que há perda de qualidade nas imagens radiológicas, porém $64,4 \%$ das respostas indicaram que a qualidade não compromete o diagnóstico. $\mathrm{O}$ sistema foi relatado como sendo fácil de ser utilizado pelos médicos (nota média de $87,8 \%$ ) e de ser assimilado para utilização (nota média de 95,6\%). Os autores sugeriram que comitês de qualidade ou conselhos padronizassem os termos e também desenvolvessem protocolos padronizados para utilização no sistema remoto e afirmaram que o envio de informações relevantes e completas seria crucial para o sucesso de uma segunda opinião médica.

Por seu turno, Silva et al. (2009) analisaram a concordância entre o diagnóstico presencial e o diagnóstico a distância (assíncrono) de lesões cutâneas, utilizando a teledermatologia. Os pacientes foram atendidos na Faculdade de Tecnologia e Ciências na cidade de Salvador - Bahia. Observou-se um grau de concordância total variando de $86,6 \%$ a $91,6 \%$. Os autores colocaram que a má qualidade da foto foi relatada em $16,6 \%$, e a necessidade de dados adicionais para uma conclusão diagnóstica foi relatada em $20 \%$.

Em outro estudo de teledermatologia, casos suspeitos de hanseníase foram encaminhados pelos médicos das clínicas para a solicitação de segunda opinião remota. Dos 106 casos utilizados, verificou-se um acordo geral de 74\% para o diagnóstico de hanseníase. A sensibilidade foi de $78 \%$, e a especificidade foi de $31 \%$. Embora com especificidade bai$\mathrm{xa}$, o estudo sugeriu que a telemedicina pode ser um método de baixo custo e útil para obter uma segunda opinião em programas de controle da hanseníase (TRINDADE et al. 2008).

Investigando a concordância entre diagnósticos dermatológicos obtidos por consulta presencial e por análise de imagens digitais a distância, Ribas et al. (2010) descrevem que 174 pacientes foram examinados por quatro dermatologistas: dois efetuaram diagnóstico presencial (A1 e A2) e dois por meio de imagens das lesões e história clínica (B1 e B2). Entre os examinadores presenciais (A1 e A2), a concordância do diagnóstico principal foi de $83,3 \%$ e, entre os de imagens (B1 e B2), de 81\%. A concordância entre o diagnóstico principal estabelecido pelo método presencial e o obtido por meio de imagens variou de $78,2 \%$ a 83,9\%, sugerindo uma concordância ótima.

Torres-Pereira et al. (2008) realizaram um estudo preliminar de teleodontologia para o diagnóstico de doenças bucais onde foram documentados 25 casos.

Cartas clínicas eletrônicas e as imagens foram produzidas e enviadas por e-mail a dois especialistas em medicina oral; estes consultores poderiam formular até duas hipóteses clínicas para cada caso. Diagnósticos finais foram comparados com os diagnósticos do atendimento remoto: em 15 casos $(60 \%)$ ambos os consultores fizeram um diagnóstico correto, em 7 casos (28\%) apenas um consultor fez um diagnóstico correto, e em 3 casos (12\%) nenhum consultor fez um diagnóstico correto. Assim, em $88 \%$ dos casos, pelo menos, um consultor foi capaz de fornecer o diagnóstico correto. Os autores indicam que a participação de dois clínicos distantes para emitir a segunda opinião melhora a taxa de diagnóstico correto. 


\section{Considerações Finais}

O desenvolvimento das Tecnologias da Informação e Comunicação bem como seu uso crescente na área de saúde têm aumentado a capacidade de formulação de sistemas de apoio para a segunda opinião especializada educacional, o que fornece oportunidade para que os clínicos, mesmo distantes de centros de referência, sejam capazes de aumentar o alcance de sua prática.

A evidência sugere que as ações de implementação do sistema contribuíram principalmente para a descentralização do atendimento, garantindo maior abrangência e acesso à saúde em lugares mais distantes, fazendo com que sejam cumpridos os direitos da população de ser atendida e indo ao encontro dos princípios e diretrizes do Sistema Único de Saúde SUS.

As questões de qualidade e fornecimento de dados insuficientes podem surgir como uma potencial preocupação na solicitação de segunda opinião através do Programa Telessaúde. Há a necessidade de padronização de termos e protocolos e a presença de ao menos dois profissionais especializados para emissão da segunda opinião de modo a prover melhor qualidade. No entanto, as iniciativas de sistemas de segunda opinião especializada parecem ser viáveis e ter boa aceitação na prática. Favoreceram o início da estruturação de redes de colaboração com um estreitamento das relações entre pesquisa (universidades) e assistência clínica (serviços).

A busca por informações referentes às possíveis aplicações da segunda opinião especializada educacional através das tecnologias da informação e comunicação mostrou o quanto essa área ainda é incipiente e o quanto pode ser explorada. Procurou-se ressaltar neste estudo as aplicações e possíveis contribuições do uso da tecnologia, sem a pretensão de detalhar cada pesquisa estudada. É recomendável que, com trabalhos futuros, publiquem-se artigos que aprofundem a discussão dos aspectos aqui abordados.

\section{REFERÊNCIAS}

BARROS, K. A. A. L.; CARDOSO, A. M. P. Avaliação da usabilidade dos sistemas de teleconsultoria médica usados na atenção primária em Belo Horizonte. Revista TEXTOS de la CiberSociedad, Monográfico: Internet, sistemas interativos e saúde, n. 16. 2008. Disponível em: <http:// www.cibersociedad.net>. Acesso em: 18 abr. 2011.

BRASIL. Portaria n.561 de 16 de março de 2006. Institui no âmbito do Ministério da Saúde a Comissão Permanente de Telessaúde. Diário Oficial da União, no ${ }^{\circ}$, Brasília, de 17 de março de 2006, Seção 1, p. 59. Disponível em: <http:// www.telessaudesp.org.br/telessaude/portaria/portaria561. aspx>. Acesso em: 07 maio 2011.

BRASIL. Portaria n. 35 de 4 de janeiro de 2007. Institui no âmbito do Ministério da Saúde o Programa Nacional de Telessaúde. Diário Oficial da União, no 4, Brasília, de 05 de janeiro de 2007, Seção 1, p. 85. Disponível em: <http:// www. telessaudesp.org.br/telessaude/portaria/portaria35. aspx>. Acesso em: 07 maio 2011.

BRASIL. Portaria n. 402 de 24 de fevereiro de 2010. Institui, em âmbito nacional, o Programa Telessaúde Brasil para apoio à Estratégia de Saúde da Família no Sistema Único de Saúde, institui o Programa Nacional de Bolsas do Telessaúde Brasil e dá outras providências. Diário Oficial da União, Brasília, 25 de fevereiro de 2010, Seção 1, p. 36. Disponível em: <http://www.telessaudesp.org.br/ telessaude/portaria/portaria 402.pdf>. Acesso em: 07 maio 2011

BRASIL. Portal telessaúde Brasil e BVS APS. Disponível em: <http://www.telessaudebrasil.org.br>. Acesso em: 18 abr. 2011.

BARBOSA, A. K., et al. HealthNet: um sistema integrado de telediagnóstico e segunda opinião médica. Grupo de Tecnologias da Informação em Saúde - LIKA, v. 5, n. 4. 2010. Disponível em: <http://www.rnp.br/wrnp2/2001/ palestras_aplicacao/res_aplic_17.pdf $>$. Acesso em: 18 set. 2011.

CARDOSO, C. S. et al. Implementation of a cardiology care program in remote areas in Brazil: influence of governability. Rural and Remote Health, v. 10, n.3, jul./ sep. 2010. Disponível em: <http:/www.rrh.org.au/articles/ subviewnew.asp?ArticleID=147 2>. Acesso em: 18 set. 2011.

HADAD, S. et al. Evaluación cualitativa de teleconsultoria del Sistema BH-Telesalud desde la Perspectiva del Médico. RevistaeSalud.com, v. 6, n. 24, e1-e15, 2010. Disponível em: <http://www.revistaesalud.com/index.php/ revistaesalud/article/view/21/35>. Acesso em: 18 set. 2011.

LIMA, C. M. A. de O.; et al. Videoconferências: sistematização e experiências em telemedicina. Radiol Bras, São Paulo, v. 40, n. 5, out. 2007. Disponível em < http:// www.scielo.br/scielo.php? script $=$ sci arttext\&pid $=$ S0100$39842007000500012 \& \operatorname{lng}=\mathrm{pt} \& \mathrm{nrm}=\mathrm{iso}>$. Acesso em: 19 jun. 2011.

MACHADO, F. S. N. et al. Utilização da telemedicina como estratégia de promoção de saúde em comunidades ribeirinhas 
da Amazônia: experiência de trabalho interdisciplinar, integrando as diretrizes do SUS. Ciência saúde coletiva, Rio de Janeiro, v. 15, n. 1, p. 247-254, jan. 2010. Disponível em: $\quad<$ http://www.scielo.br/ scielo.php?script=sci arttext\&pid=S1413-81232010000100030\&lng=en\&nrm $=\overline{\mathrm{i}}$ so>. Acesso em: 26 set. 2011.

MIOT, H. A.; PAIXÃO, M. P.; WEN, C. L. Teledermatologia: passado, presente e futuro. An Bras Dermatol, Rio de Janeiro, v. 80, n. 5, p. 523-532, oct. 2005. Disponível em: $\quad<$ http://www.scielo.br/scielo.php?script $=$ sci arttext\&pid=S0365-05962005000600011\&lng=en\&nrm $=\overline{\mathrm{i}}$ so>. Acesso em: 26 set. 2011.

MONTEIRO, L. F., et al. Segunda Opinião Formativa via Eletrocardiografia Digital e Informações Clínicas de um Sistema de Registro Eletrônico em Saúde. In: V Congresso Sul Brasileiro de Computação [recurso eletrônico]; IV Workshop de Trabalhos Acadêmicos; I Workshop de Inteligência Computacional Aplicada, 2010, Criciúma. Anais eletrônicos... Criciúma: UNESC, 2010. Disponível em: <http://periodicos.unesc.net/index.php/sulcomp/article/ viewFile/265/271>. Acesso em: 26 set. 2011.

NEIRA, R. A. Q. et al. Avaliação de um sistema de segunda opinião em radiologia. Radiol Bras, São Paulo, v. 43, n. 3, p. 179-183, june 2010. Disponível em: <http://www. scielo.br/scielo.php?script $=$ sci_arttext\&pid $=$ S0100$39842010000300010 \& \operatorname{lng}=\mathrm{en} \& n \mathrm{~nm}=\mathrm{iso}>$. Acesso em: 26 set. 2011.

ORGANIZAÇÃO MUNDIAL DE SAÚDE. Tecnologia da informação de apoio à saúde. Disponível em: <http://www. who.int/eht/en/InformationTech.pdf $>$. Acesso em: 26 abr. 2011.

RIBAS, J. et al. Concordância entre diagnósticos dermatológicos obtidos por consulta presencial e por análise de imagens digitais. An Bras Dermatol, Rio de Janeiro, v. 85, n. 4, aug. 2010. Disponível em: <http:// www.scielo.br/scielo.php?script=sci_arttext\&p id= S036505962010000400004\&lng=en\&nrm=iso $>$. Acesso em: 26 set. 2011.

RIBEIRO, A. L. P. et al. Implementation of a telecardiology system in the state of Minas Gerais: the Minas Telecardio Project. Arq Bras Cardiol, São Paulo, v. 95, n. 1, july 2010. Disponível em: <http://www. scielo.br/scielo.php?script $=$ sci_arttext \&pid=S0066-782 $\mathrm{X} 2010001100011 \& \operatorname{lng}=\mathrm{en} \& \mathrm{nrm}=\mathrm{iso}>$. Acesso em: 26 set. 2011.

RIBEIRO FILHO, J. L. et al. Telemedicina e telessaúde a construção de redes colaborativas de ensino, pesquisa e assistência ao diagnóstico e ao tratamento. Informática Pública, v. 10, n. 2, p. 97-104, 2008.

SILVA, C. S. et al. Teledermatologia: correlação diagnóstica em serviço primário de saúde. An Bras Dermatol, Rio de Janeiro, v. 84, n. 5, oct. 2009. Disponível em: <http://
www.scielo.br/scielo.php?script=sci_arttext\&pid=S036505962009000500007\&lng=en\&nrm=iso >. Acesso em: 26 set. 2011.

SOIREFMANN, M. et al. Telemedicina: uma revisão da literatura. Rev HCPA, Porto Alegre, v. 28, n. 2, p. 116-119, 2008 .

TIS - Grupo de Tecnologias da Informação em Saúde. Tele o quê? Aplicações. Recife, 2011. Disponível em: <http:// www.telesaude.org.br/oquee1.htm>. Acesso em: 18 jun. 2011.

TRINDADE, M. A. B. et al. Accuracy of store-and-forward diagnosis in leprosy. J Telemed Telecare. v. 14, n. 4, p. 208210 , june 2008 .

TORRES-PEREIRA, C. et al. Email for distance diagnosis of oral diseases: a preliminary study of teledentistry. J Telemed Telecare, v. 14, p. 435-438, dec. 2008.

WALDMAN, E. A. Vigilância em saúde pública. São Paulo: Faculdade de Saúde Pública da Universidade de São Paulo, 1998. 253 p. (Série Saúde \& Cidadania, v. 7).

WEN, C. L. Telemedicina e telessaúde - um panorama no Brasil. Informática Pública, Belo Horizonte, v. 10, n. 2, p. 07-15, dez. 2008. Disponível em: <http:/www.ip.pbh.gov. br/ANO10_N2_PDF/telemedicina_telesaude.pdf $>$. Acesso em: 18 abr. 2011.

.; HADDAD, A. E. Telemática e telemedicina em apoio à atenção primária no Brasil: Ação nacional é parceria entre ministérios da saúde e da ciência e tecnologia. Disponível em: <http://www.telessaudesp.org.br/telessaude/ artigo.aspx\#>. Acesso em: 18 abr. 2011.

Segunda Opinião Especializada Educacional. Telessaúde Núcleo São Paulo. Disponível em: <http:// www.telessaudesp.org.br/telessaude/SegundaOpiniao 2.aspx>. Acesso em: 18 abr. 2011. 